www.jmscr.igmpublication.org

ISSN (e)-2347-176x ISSN (p) 2455-0450

crossrefDOI: https://dx.doi.org/10.18535/jmscr/v7i1.85

Journal Of Medical Science And Clinical Research

IGM Publication

An official Publication of IGM Publication

\title{
Effectiveness of Laparoscopic Evaluation of Women with Previous Endometriosis Surgery for Improving Fertility
}

Authors

\section{Dr Shamsun Nahar ${ }^{1}$, Dr Dilshad Jahan ${ }^{2}$, Dr Naznin Akther ${ }^{3}$, Dr Sk. Farid Uddin Ahmed $^{4}$, Dr Dilip Kumar Kundu ${ }^{5}$}

${ }^{1}$ Professor \& Head, Department of Obstetrics and Gynecology, Khulna Medical College, Khulna, Bangladesh

${ }^{2}$ Medical Officer (Private), Bangladesh

${ }^{3}$ Assistant Professor, Department of Obstetrics and Gynecology, Gazi Medical Collage Hospital, Khulna

${ }^{4}$ Associate Professor, Department of Anesthesiology, Khulna Medical College, Khulna, Bangladesh

${ }^{5}$ Assistant Professor (Anesthesiology), Khulna Medical College, Khulna, Bangladesh

*Corresponding Author

Prof. Dr Shamsun Nahar

Email: gynaekmc@gmail.com, Mobile \# +8801711430888

\begin{abstract}
Background: Endometriosis is a common and important health problem of women. This exact prevalence is unknown because surgery is required for it's diagnosis. Endometriosis is more prevalent in the setting of infertility, with proper patient selection, a meticulously preformed laparoscopic, surgery is an excellent option that provides these patients to achieve repeated future pregnancies.

Objective: Laparoscopic evaluation of previous open or laparoscopy surgery in women with all stage of endometriosis for improving fertility.

Methods: Khulna medical college (KMC) and Victory endoscopic center based cross sectional descriptive study was performed in 2008 - 2016 in 200 cases for laparoscopic assessment and management of pelvic endometriosis with infertility. Criteria for performing laparoscopy was as follows: after initial laparoscopy or open surgery no improvement of pelvic pain, no improvement of fertility or no improvement of dysmenorhoea, dyspareunia and/or recurrence of chocolate cyst. Exclusion criteria's were age above 40 years and Low AMH or ovarian reserve.

Results: Within 200 cases, the age ranges from 25 - 40 years and period of infertility less then 3 years about $39 \%$ and more than $61 \%$. Primary infertility was $85 \%$ and secondly infertility was $15 \%$. Within 200 cases open surgery were 109 and laparoscopy were 91 cases and the documentation of previous surgeries were stage I endometriosis, $5.5 \%$, stage - II 26.5\%, stage III 33\% and stage - IV 19.5\%, documentation was lost in $15.5 \%$. The surgeries which we had done reconstinction of pelvic anatomy and cystectomy but 8 patients were quired total abdominal hysterectomy and bilateral salphingeatomy due to frozen pelvis. 11 patient's were referred to IVF and ET centre. Within these cases our achieved pregnancy rate was about $31 \%$ \& rest are under observation.

Conclusion: Relaparoscopy gives beneficial result if in young patient with good ovarian reserve. IVF treatment is definitely a suitable option for older patients and after $1^{\text {st }}$ time surgery. But exclusion of age and ovarian reserve, $2^{\text {nd }}$ time laparoscopic management is beneficial where limited facilities for IVF and ET.

Keywords: Previous endometriosis surgery, laparoscopy, infertility.
\end{abstract}




\section{Introduction}

Endometriosis is a benign disease of genital tract in which abnormal growths of tissue histologically resembling the endometriotic like tissue, consisting of glands and stroma found outside the endometrium. Although implanted ectopically, this tissue presents histopathological and physiological responses that are similar to the responses of the endometrium. Incidence and Prevalence are increasing significantly ranges from $1 \sim 50 \%, 1 \sim 2 \%$ in general population, $30 \sim 50 \%$ in Infertile women, typically diagnosed in women within 25-35 years age $\mathrm{e}^{\mathbf{1 , 2}}$. Diagnosed in approximately $45 \%$ of women undergoing laparoscopy in infertility \& $35 \%$ with complaint of chronic pelvic pain. Family history increases risk ten-fold \& significant cause of morbidity. It's a "Disease of theories"; definitive cause or causes remain under debate, though associations with number of hereditary, environmental, genetic, and menstrual characteristics exists. The diagnostic hypothesis of endometriosis is based on the clinical history, along with the results from gynecological examinations, laboratory tests and transvaginal ultrasound. Diagnosis by means of laparoscopy, which is considered the gold standard, some clinical characteristics, the physical examination itself, laboratory test results and evidence from imaging examinations may suggest the diagnosis. The greatest difficulty lies in diagnosing minimal and mild lesions. In these cases, the ideal access for confirmation is always laparoscopic, since the complementary examinations available do not offer the necessary specificity. The Chinese guidelines for the diagnosis and treatment of endometriosis-related infertility allow couples to have a period of about 12 months for potential spontaneously conception after surgery before being offered IVF-ET, especially in younger women with minimal or mild endometriosis. ${ }^{3}$ The endometriosis fertility index (EFI) has a predictive value for pregnancy after surgery. In vitro fertilization and embryo transfer (IVF-ET) is a good treatment of infertility. This study aimed to reassessment the factors affecting the spontaneous pregnancy, who had done previous laparoscopy or open surgery for management of endometriosis related infertility.

\section{Materials and Methods}

This was a retrospective cross sectional study was conducted in Victory Endoscopic Center and obs \& gynae department of $\mathrm{KMCH}$. We selected 200 cases for laparoscopic assessment and management of pelvic endometriosis with infertility from the period of January 2008 - 2016, who had not achieved pregnancy within 3 years after initial laparoscopy surgery due to pelvic endometriosis or endometrioma. The collated data included age, parity, history of infertility, mode of surgery and subsequent fertility for all endometriosis with infertility patient's.

Criteria for performing laparoscopy was as follows: after initial laparoscopy or open surgery, no improvement of pelvic pain, no improvement of fertility no improvement of dysmenorhoea or dyspareunia and requrrence of chocolate cyst.

The patient should be reassessed clinically and by investigations that was transvaginal sonography scan, which helped to determined ovarian reserve, number of antral follicles or follicular maturation, sono tenderness, pelvic mass or blood in pelvis. Assessment of CA 125 level, taking laparoscopy biopsy form endometrial lesion. After initial laparoscopy or open surgery AMH test had done for detection of poor ovarian reserve in suspected cases.

Exclusion criteria's were age above 40 years and Low AMH or ovarian reserve. Under general anesthesia pneumo peritoneum was made by $\mathrm{CO}_{2}$. After introduction of telescope total assessment of pelvic cavity was done followed by adhesiolysis and restoration of pelvic anatomy, chocolate cystectomy and tubal patcncy test. After surgery, the patient's were advised to actively attempt to conceive, either naturally or using ovulation inducing drugs or with IVF-ET depends upon to surgical correction and ovarian reserve. 


\section{Results}

We had done 200 cases of laparoscopic surgery from the period of January 2008 to January 2016, in victory endoscopic centre and KMC, Who had not achieved pregnancy within 3 years after initial open / laparoscopic surgery due to pelvic endometriosis or endometrioma. After completion of laparoscopic surgery in different stage of disease, all cases were follow up at least 1 year. Age ranges of this patients were 25-40 years. Table -1 presented the patient age, period of infertility and history of previous pregnancy. Within these 200 cases open surgeries were 109 and laparoscopic surgeries were 91 cases, showing in figer I. Initial laparoscopy / open - surgery for Endometriosis Stage I - 11(5.5\%), Stage 11 - 53 (26.5\%) Stage 111 - 66 (33\%), Stage IV - 39 (19.5\%) Inadequate or no documentation - 31 (15.5\%), showing in figer - II. Again laparoscopic evaluation was done in 200 cases and findings was plotted in table - II. Table - III was presented the surgery which we had done.

Table-1 Characteristics of the women included in the study (n-200)

A.

\begin{tabular}{|l|c|}
\hline Characteristics & Values \\
\hline Age (year) & $(20-38$ years $)$ \\
\hline$>35$ & $4 \%$ \\
\hline $30-35$ & $46 \%$ \\
\hline $25-29$ & $43 \%$ \\
\hline $20-24$ & $7 \%$ \\
\hline
\end{tabular}

B. Infertility length in year.

C. Previous Pregnancy

\begin{tabular}{|l|l|}
\hline$<3$ years & $39 \%$ \\
\hline$>3$ years & $61 \%$ \\
\hline
\end{tabular}

\begin{tabular}{|l|l|}
\hline Yes & $15 \%$ \\
\hline No & $85 \%$ \\
\hline
\end{tabular}

The following Figure-1 showing number of initial open and laparoscopic surgery

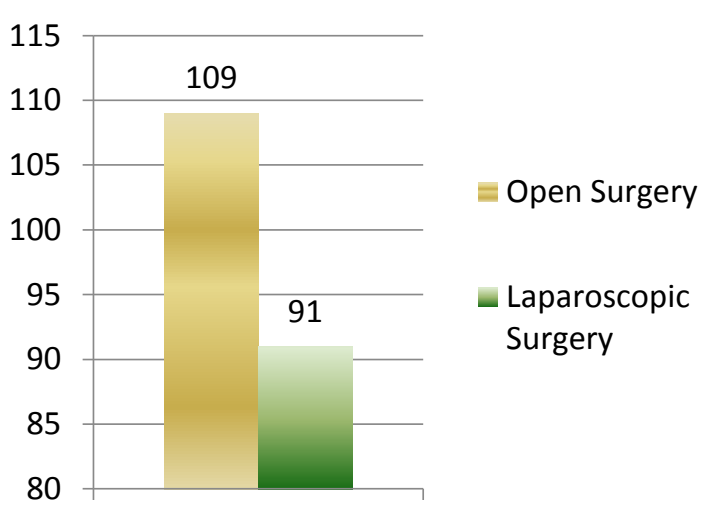

Figure1: initial open and laparoscopic surgery

The following Figure-2 showing the findings of initial laparoscopy /open - surgery for endometriosis

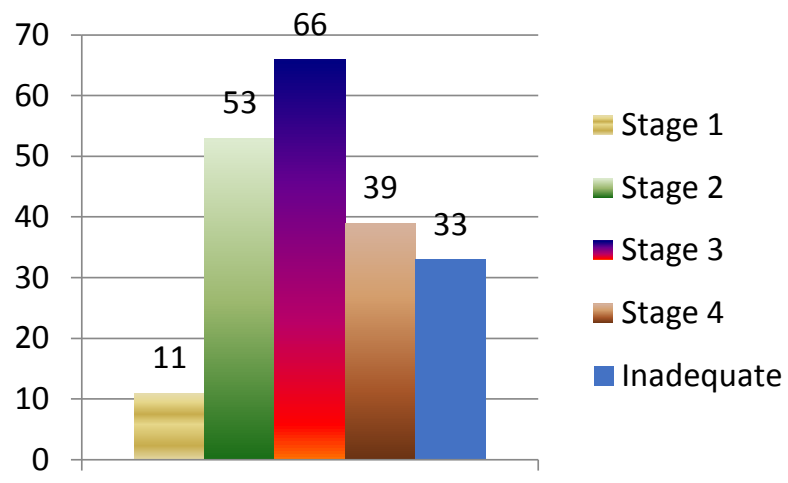

Figure-2: Initial laparoscopy /open - surgery for endometriosis

Laparoscopic findings showing in table- 2

Table-2: Laparoscopic findings

\begin{tabular}{|l|c|}
\hline Normal pelvic anatomy & $17(8.5 \%)$ \\
\hline Mild endometriosis bilateral & $\begin{array}{c}25(12.5 \%) \& 48(24 \%) \\
\text { respectively }\end{array}$ \\
\hline $\begin{array}{l}\text { Unilateral \& bilateral } \\
\text { adhesion }\end{array}$ & $46(23 \%), 20(10 \%)$, \\
\hline $\begin{array}{l}\text { Unilateral \& } \\
\text { endometrioma }\end{array}$ & $17(8.5 \%)$. \\
\hline $\begin{array}{l}\text { Short tube tubal adhesion } \\
\text { and fimbrial agglutination }\end{array}$ & \\
\hline
\end{tabular}

The surgeries which had done showing in table-3

Table-3: Surgeries done

\begin{tabular}{|c|c|}
\hline $\begin{array}{l}\text { Open the blocked tubes and/ or } \\
\text { restore the normal pelvic } \\
\text { anatomy } \\
\text { One Tube } \\
\text { Both } \\
\text { Adhesiolysis }\end{array}$ & $\begin{array}{l}92 \text { cases } \\
17 \\
6 \\
85\end{array}$ \\
\hline $\begin{array}{l}\text { Cystectomy and reconstruction } \\
\text { of ovary }\end{array}$ & 70 cases. \\
\hline Normal findings were & 17 cases. \\
\hline Conversion to laparatomy & 8 cases. \\
\hline
\end{tabular}


Conversion from laparoscopy to laparatomy followed by total abdominal hysterectomy and bilateral salpingophrectomy were in 8 , due to frozen pelvic, adenomyosis and hydrosalpinx. One case had developed pelvic collection after laparoscopic which was drained by colpotopy, another one was directly trocar injury to the small intestine due to previous midline incision with dense intestinal adhesion. Advice for IVF and ET in bilateral blocked tube in 11 cases. After laparoscopic surgery, clomiphene / letrol was used in 168 cases. Within one to three year follow up, 59 cases achieved pregnancy, abortion 2, 11 patients were advised for assisted reproduction and rest are under observation.

\section{Discussion}

Endometriosis is usually a multifocal progressive disease tends to come back after treatment and lasts as long as there is ovarian function, that is until menopauses. Therefore, prompt definitive diagnosis and staging are extremely important for lifelong treatment, recurrence prevention and fertility improvment. The first grossly recognizable lesions are blister like blebs $2-3 \mathrm{~mm}$ in diameter on the surfaces of target organs. Over time, these blebs assume the characteristic powder burn" appearance produced by a collection of hemorrhagic spots and bluish-red nodules or patches. The lesions may become cystic, but the cysts do not enlarge except in the ovary. As the disease progresses, the endometriotic sites may become fibrotic, distorting the affected organ and forming adhesions with the surrounding structures. The ovaries may be affected in two ways. Small implants of endometrial tissue may cause paraovarian scarring and adhesions, or they may evolve into endometriomas or chocolate cysts. Endometriomas are moderately thin-walled cysts containing dark, semisolid, sticky material that represents hemorrhage within the lumen of the cyst. Although the chocolate cyst is highly suggestive of endometriosis, hemorrhage within a corpus luteum cyst, a follicular cyst, or even a neoplastic cyst can have a similar appearance.
When the uterine ligaments are involved, especially the uterosacral ligaments, thickening of these structures occurs due to endometriotic nodule formation, which tenders the ligaments. The standard of reference for the diagnosis and staging of endometriosis is laparoscopy but sometimes the presence of endometriosis is observed by overlying dense adhesion, if carful dissection is performed ${ }^{4}$. Serum CA-125, MR imaging is helpful for in patients with known endometriosis who present with recurrent pain, MRI helps to visualize laparoscopy blind spots beneath dense adhesions and at extra peritoneal sites such as the rectum, the vagina or within the bladder. ${ }^{5,6}$ Options for endometriosis infertily are surgery, IVF-ET, Oocyte donation, Oocyte cryopreservation before surgery. The aim of this surgery is to 1. destroy or remove areas of endometriosis 2. destroy or remove ovarian endometriotic cysts, by cutting away or excising the cyst wall (capsule) or opening the cyst, draining the chocolate fluid and destroying the capsule 3. divide adhesions to mace free tissues for improve fertility. The surgeon should also be proposed to reseal endometiotic lesions that may involve other organs such as the bowel or bladder. Appendiectomy should also be performed if there any adhesion or if endometriosis involves the appendix. Currently, the main choice for the diagnosis and treatment of endometriosis is laparoscopic surgery. ${ }^{6}$ For infertile women with endometriosis as the only identifiable infertility factor, operative laparoscopy can improve pregnancy rates (PRs) in all stages. ${ }^{7}$ For women with minimal-to-mild endometriosis, operative laparoscopy is more effective than diagnostic laparoscopy in improving the pregnancy rate. ${ }^{7,8,9}$ For women with moderate and severe endometriosis, the spontaneous PR after laparoscopic surgery is much higher compared with those treated by expectant management. ${ }^{10}$ The conception after surgery was 59\% (4), which reconfirmed the effectiveness of laparoscopic surgery. There 8 patients were required complete removal of uterus and both ovaries by laparatomy, 
due to destruction of total anatomy by dense adhesion associated with adenomyosis. Success rates of laparoscopy surgery are reported and studies suggest a $62.57 \%$ improvement or regulation of pain at 6 months, with $55 \%$ still improved at 12 months.

The recurrence rate of endometriosis is unpredictable, but is generally reported to be in the range of $5-20 \%$ per year ${ }^{11}$. Women wishing to get pregnant should start trying as soon as possible after surgery. Sometime recurrence may be related to incomplete removal or destruction of the disease or if endometriosis is severe and affecting organs such as bowel, it may not always be possible to remove all of the endometriosis during one laparoscopy. The cysts are attached to the other structures by plaques of endometriosis, the plaques should be removed with cyst at the same time, otherwise it may confine and caring pain and adhesions. Detection of recurrence is suspected on the women history of very painful menstrual cycle, painful intercourse or frozen pelvis by per vaginal examination or ultrasound finding, CA-125 level or MR imaging.

Before going to invasive procedure observed ovarian reserve carefully. Those have a low AMH and age above $>40$ years should consider here for IVF and ET with donor ovum. Our selected patients were 200 but about $31 \%$ women missed their previous surgical documents.

The impact of surgery for ovarian endometriomas on ovarian reserve has been a subject of great interest. Poor response to ovarian stimulation for assisted reproduction has been reported in patients who had undergone excision of ovarian endometrioma. ${ }^{12}$ A younger age at menopause and premature ovarian failure has also been reported respectively ${ }^{13}$. Typically, AMH has been used as the marker for reduced ovarian reserve in these studies in two systematic views performed a metaanalysis to investigate the impact of surgery for endometriomas on ovarian reserve as determined by serum anti-müllerian hormone (AMH). ${ }^{14,15}$

Antral follicle is a reliable marker for ovarian reserve and has the advantage of correlating with the ovarian reserve of a single ovary, concluded the ovarian reserve evaluated with AFC was not significantly impaired by surgical excision of the ovarian endometrioma when it was performed with the stripping technique. ${ }^{16}$ another study reported a nine-month cumulative pregnancy rate of $24.4 \%$ in 18 women undergoing second surgery for moderate or severe endometriosis (Stage III or IV) and a pregnancy rate of $33.3 \%$ and $69.6 \%{ }^{17}$ but in our group $31 \%$ achieved pregnancy and rest are under observation. Major disadvantages of surgery are cost, morbidity, and longer time to conception. IVF treatment is definitely a suitable option for older patients, and there are no scope for improving by using ovulation induction or intrauterine insemination (IUI)

\section{Conclusion}

Recurrence of pelvic endometriosis after surgery is common in reproductive period. Diagnosis is dependent on the clinical consideration of the possibility of the disease, physical examination and a good reception of medical history associated with TV scan, MRI and histopathology examination. Again laparoscopic evaluation is beneficial and facilitated the way of natural fertility but elderly patients need to careful evaluation about ovarian reserve and better option for IVF and ET. This study could not only evaluate the optimal time to propose IVF treatment after surgery, but also reassessment and management - definitely improve fertility after proper selection of patients. Re-evaluation information can be done by patients and physicals as a reference to determine when to adopt IVFET.

\section{References}

1. Houston DE. Evidence for the risk of pelvic endometriosis by age, race and socioeconomic status. Epidemiol Rev. 1984;6:167-91.

2. Jansen RP, Russell P. Nonpigmented endometriosis: clinical, laparoscopic, and 
pathologic definition. Am J Obstet Gynecol. 1986; 155(6):1154-9.

3. Cooperative Group of Endometriosis, Chinese Society of Obstetrics and Gynecology, Chinese Medical Association. Guideline for the diagnosis and treatment of endometriosis (in Chinese) Chin J Obstetr Gynecol. 2015;50:161-9. Doi: 10.3760/cma.j.issn.0529-567x.2015.03.001. [PubMed]

4. Anderson MC. Endometriosis. In: Symmeo WSTC, ed. Systemic pathology. 3rd ed, vol 6. New York, NY: Churchill Livingstone, 1991; 263-275.

5. Dan H, Limin F. Laparoscopic ovarian cystectomy versus fenestration/coagulation or laser vaporization for the treatment of endometriomas: A meta-analysis of randomized controlled trials. Gynecol Obstet Invest. 2013;76:75-82. doi: 10.1159/000351165. [PubMed]

6. Togahi K, Niohiomra K, Kimula I etalEndometrial cyst: diagrosis of MR imaging. Radiology 1991; 180:73-78.

7. Jacobson TZ, Duffy JM, Barlow D, Farquhar C, Koninckx PR, Olive D. Laparoscopic surgery for subfertility associated with endometriosis. Cochrane Database Syst Rev. 2010;1:CD001398. doi: 10.1002/14651858. [PubMed]

8. Practice Committee of the American Society for Reproductive Medicine. Endometriosis and infertility: A committee opinion. Fertil Steril. 2012;98:1400-6. doi: 10.1016/j.fertnstert.2012.05.031. [PubMed]

9. Dunselman GA, Vermeulen N, Becker C, Calhaz-Jorge C, D'Hooghe T, De Bie B, et al. ESHRE guideline: Management of women with endometriosis. Hum Reprod. 2014;29:400-12.

doi:

10.1093/humrep/det457. [PubMed]

10. Vercellini P, Fedele L, Aimi G, De Giorgi
$\mathrm{O}$, Consonni
D, Crosignani
PG.

Reproductive performance, pain recurrence and disease relapse after conservative surgical treatment for endometriosis: The predictive value of the current classification system. Hum Reprod. 2006;21:2679-85. doi: 10.1093/humrep/del230. [PubMed]

11. Winkel CA. Evaluation and management of women with endometriosis. Obstet Gynecol 2003;102: 397- 408

12. Hart RJ, Hickey M, Maouris P, et al. Excisional surgery versus ablative surgery for ovarian endometriomata. Cochrane Database Syst Rev. 2008;(CD004992) [PubMed]

13. Coccia ME, Rizzello F, Mariani G, et al. Impact of endometriosis on in vitro fertilization and embryo transfer cycles in young women: a stage-dependent interference. Acta Obstet Gynecol Scand. 2011;90:1232-1238. [PubMed]

14. Raffi F, Metwally M, Amer S. The impact of excision of ovarian endometrioma on ovarian reserve: a systematic review and meta-analysis. J Clin Endocrinol Metab. 2012;97:3146-3154. [PubMed]

15. Somigliana E, Benaglia L, Vigano $\mathrm{P}$, et al. Surgical measures for endometriosis-related infertility: a plea for research. Placenta. 2011;32(Suppl. 3):S238-S242. [PubMed]

16. Muzii L, Di Tucci C, Di Feliciantonio M, et al. The effect of surgery for endometrioma on ovarian reserve evaluated by antral follicle count: a systematic review and meta-analysis. Hum Reprod. 2014;29:2190 2198. [PubMed]

17. Pagidas K, Falcone T, Hemmings R, et al. Comparison of reoperation for moderate (stage III) and severe (stage IV) endometriosis-related infertility with in vitro fertilization-embryo transfer. Fertil Steril. 1996;65:791-795. [PubMed] 